\title{
The Safety Impact of Additional Blue Lights of Rescue Vehicles
}

\section{Andreas Leich ${ }^{a}$, Hagen Saul $^{a}$, Ragna Hoffmann ${ }^{a}$, Karsten Kozempel ${ }^{a}$, Andreas Luber $^{a}$, Uwe Kippnich ${ }^{b}$, Markus Damböck ${ }^{b}$, Rainer Rauschenberger ${ }^{b}$, Florian Biber $^{b}$, Thomas Stadler ${ }^{b}$}

${ }^{a}$ German Aerospace Center, Institute of Transportation Systems, Rutherfordstr. 2, 12489 Berlin, Germany, andreas.leich@dlr.de ${ }^{b}$ Bavarian Red Cross (BRK) Headquarters, Garmischer Str. 19-21, 81373 München

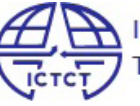

international Co-operation on

Theories and Concepts in Traffic Safety

This paper was presented at ICTCT workshop.
KEYWORDS: Ambulance Vehicles, Traffic Safety, Risk, additional flash lights (3 - 5 keywords)

\section{BACKGROUND}

The Bavarian Red Cross (BRK) aims to increase the safety of ambulance vehicles, so called Mobile Intensive Care Units (MICU) in road traffic. One possible measure in this context is to improve the visibility of MICU through additional side flashing blue lights. In Figure $1 \mathrm{a}$ MICU is shown with additional side blue lights.

The additional side blue lights aim to increase the perception of other vehicle drivers of a MICU that is entering the road from the side. This seems to be reasonable, especially, when the MICU is suddenly appearing from behind a building or from a narrow intersecting road. This approach, although intuitive, has raised discussion between the BRK and the public authorities responsible for type approval. In order to not overstrain road users and minimize special signaling in public space, authorities need to have proof of significant positive effects of these additional lights. A literature review revealed no clear answer. Therefore, a quantitative analysis on the positive effects of the proposed measure was needed.

The aim of the study presented in this paper was to conduct efficacy studies of additional side blue lights in road traffic based on empirical data.

\section{AIMS AND METHODS}

\subsection{Related Work}

The high crash risk particular during emergency drives has been reported in numerous studies (see e.g. Casey, 2011). 

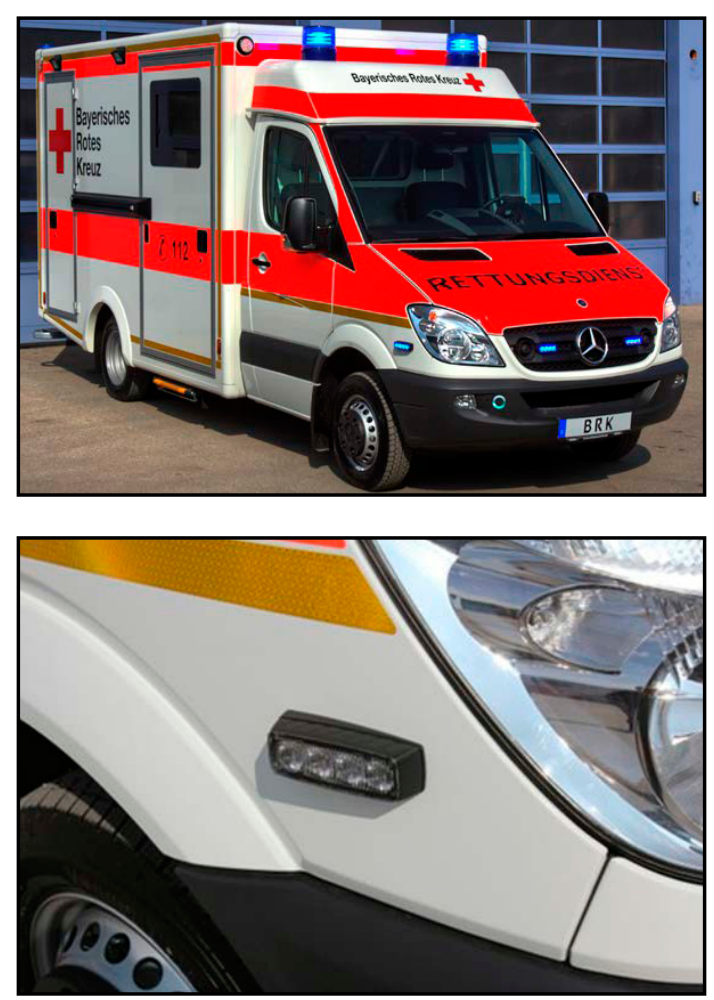

Figure 1. Additional side mounted flashing blue lights at a MICU (source: BRK)

Regarding the safe operation of emergency vehicles numerous best practice papers exist. Boone et al. (2014) summarizes best practice information received and consolidates it in an appendix for consideration by the emergency medical services community. Information on case studies of past tragedies, loss statistics, organizational and personal responsibilities and strategies to improve fire fighter and law enforcement officer safety while in vehicles is published by the International Association of Fire Fighters (2010). This document contains as well information on emergency vehicle lighting and markings and on safety while working at the road side. However, specific answers to the question raised by type approval authority could not be found in the literature.

Real accidents are rare and difficult to observe events. For this reason surrogate safety measures were introduced and studied in the literature. Gettman et al. (2008) assessed the correlation between conflicts recorded by a surrogate safety assessment model (SSAM) and actual crash histories at 83 urban intersections. Regression was used to establish the following peak-hour conflict-based model to predict average annual intersection crash frequency. This equation exhibited a correlation (R-squared value) of 0.41 which was in line with previous studies for urban signalized intersections. The evaluation of Guo et al.
(2010) based on data collected by natural driving studies supports the assumption that there is a strong relationship between the frequency of crashes and near-crashes. Souleyrette and Hochstein (2012) tried to apply the correlation formula of Gettman et al. (2008) and did not succeed well. This supports the statement of Guo et al. (2010) that the frequency relationship between accidents and near crashes is evident but complex and may depend on numerous factors. Thus, it was concluded, that a comparison of the frequency of critical situations depending on the presence of additional blue lights should give a good qualitative forecast on the expected frequency of real accidents. Therefore, a surrogate safety measure study was found to be appropriate to address the task.

However, the following potential pitfalls were identified:

a) Caution is needed when generalizing from the findings on one research location to conclusions for the whole road network.

b) Quantitative forecast is to be faced with caution.

c) Critical situations with MICU involved are rarer events than critical situations in normal traffic.

These pitfalls in mind, the experimental approach was laid out.

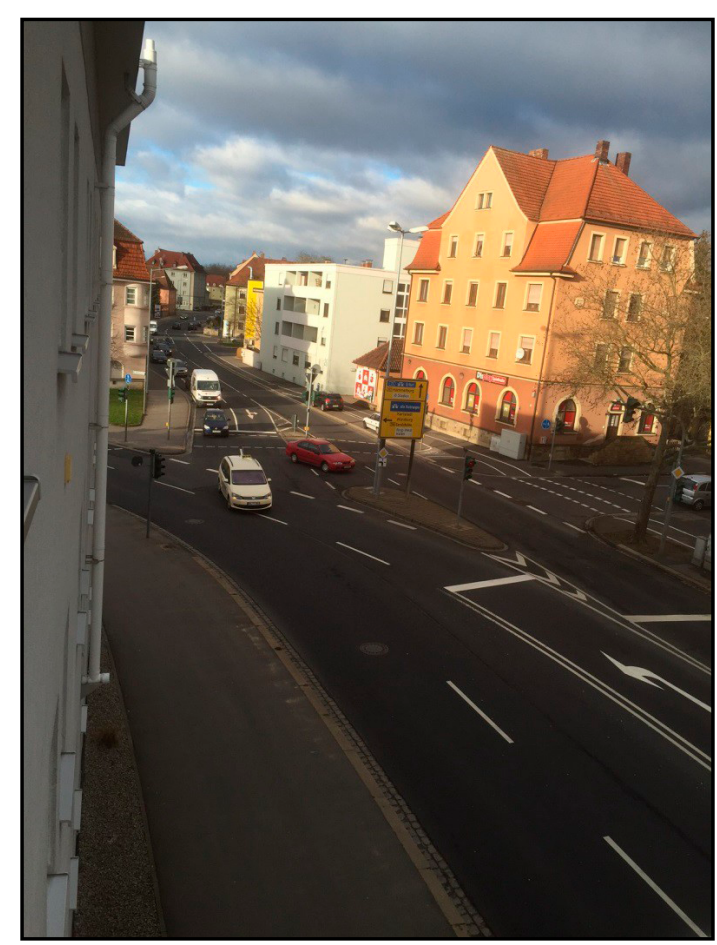

Figure 2. Picture of the location BRK Kreisverband Schweinfurt, rescue station Schweinfurt, Niederwerrner Str. 13, looking west (Friedrich-Ebert-Straße) 


\subsection{Approach}

The goal of the study is to provide a principal feasibility of a proof with comparatively little effort. The effectiveness of the additional side blue lights is therefore estimated based on comparative examinations in which two vehicle classes participate: vehicles with and without additional flashing blue lights. By means of image processing methods it is examined, how often critical situations occur in flowing traffic, when an MICU enters the free-flowing traffic. Critical situations can generally be assessed using criticality measures such as TTC (time to collision), PET (near miss), DRAC (necessary deceleration to prevent an accident) and location of braking points, as well as the maximum deceleration. It is a common approach using video surveillance for recording road user trajectories and automatically identifying potential critical situations (Ismail, 2010).

The approach of the study was to record digital videos of the traffic on the research site, to evaluate the video material afterwards using digital image processing methods and to determine the distributions of the location and frequency of critical situations. Based on these distributions safety promoting measures and installations can be evaluated and compared. Distributions of the following standard parameters (Fitzpatrick, 2000) were used:

- Maximum deceleration of vehicle.

- Entry speed in the region of interest.

- Braking point.

- Point when walking speed is reached.
The evaluation was conducted with anonymized data: digital videos had been recorded with low resolution to not contain personally identifiable data and safety measures have been calculated on anonymized trajectories.

The practical steps were as follows: First of all, still photo test images were requested by various rescue teams in order to be able to assess the suitability of the site for a comparative examination. The rescue station Schweinfurt in Niederwerrner Straße 13 (see Figure 2) was selected. Video recordings of the traffic situation in front of the BRK rescue station in Schweinfurt were created in order to assess the feasibility of the approach.

Afterwards, an on-site appointment took place in the rescue station Schweinfurt, where test pictures were taken from different office windows in order to find the suitable perspective (see example at Figure 2). Finally, a perspective was selected that is characterized as follows (Figure 3):

- Unobstructed view of the traffic approaching the exit of the rescue station.

- The vehicles on Niederwerner Straße pass the exit of the rescue station

- Outward MICU cross the carriageway from the right

- Outward MICU emerge from behind a house, i.e. they are completely or partially hidden before they hit the road

- The road users can be detected within a range of $35 \mathrm{~m}$ from the potential point of conflict and
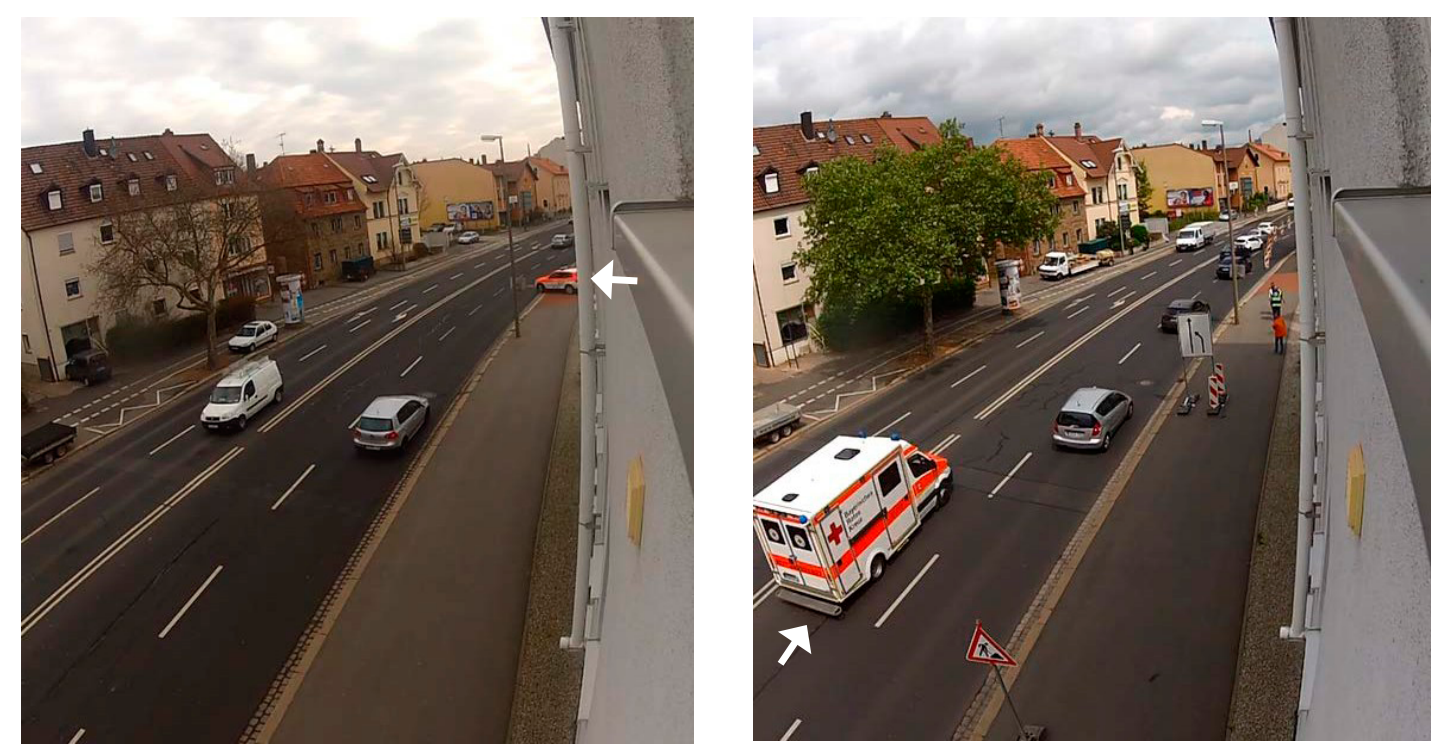

Figure 3. Picture of detection area and interacting vehicles (East view). Left: Interaction with MICU coming from the south, Right: Interaction with MICU when approaching from the west 
their movement lines (trajectories) recorded in order to detect the possible interaction between MICU and the road users

Then, a commercial camera (GoPro Hero II) was placed in the window of one of the ambulance offices (Figure 3). This recorded a digital video over a period of 4-5 hours. Afterwards it needed to be recharged. In total 14 days of videos were recorded.

The inspection of the recorded material showed that within the 14 days a total of 51 missions with siren and blue light had been recorded. Of these, 13 scenes were containing vehicles with side blue lights in use and 38 scenes without side blue lights. A closer examination of the scenes revealed the following:

- Often there is no vehicle interaction between MICU and other road users because there is no traffic. Those were discarded.

- Several recorded operations from East and West were carried out by other rescue services, like police and fire brigade. These were discarded as well.

- Construction work or marking work on the road took place on several days.

- Operation was started in different traffic conditions. The spectrum ranges from free flow traffic to traffic jams.

- In a single scene, two MICU appeared immediately one after the other: one with and one without side blue lights.

- In two scenes the MICU approached from the west (see Figure 3 on the right), in all other scenes the MICU left the station coming from the south via the exit (Figure 3).

The study was carried out with software tools for the detection and tracking of road users in video sequences, which were developed especially for this purpose at the German Aerospace Center (Leich et al., 2015). The software is capable of recognizing vehicles in pre-defined detection areas (virtual loops) and of tracking them through the area of sight. In contrast to other publicly available tools, the software is able to continue with the tracking task when the viewing axis of the camera is flat and there is much occlusion between road users present in the scene. The application of this software makes it necessary to conduct the following steps:
- Provide video scenes

- Determine the parameters of the exterior and interior orientation of the camera for the various 14 days of use.

- This model of optical imaging allows a projection of the action in the two-dimensional camera image onto the real 3-D world; thus speeds etc. become measurable

- Provide training data for the virtual loops

- Automatic processing of video sections, generation of trajectories

- Evaluate the trajectory data and collect of the relevant parameters described above

\subsection{Determination of the exterior orientation parameters}

At each of the 14 different shooting days, the camera was mounted in the same position, but there were slight variations in orientation. These arise when the camera is re-inserted manually into the holder, which leads to minimal changes in the orientation of the camera. These minimal changes result in errors of several meters when projecting image coordinates into world (UTM) coordinates. The projection error increases with distance from the camera. As stated before, the projection is necessary in order to derive trajectories in world coordinates and measuring position and kinematics in common metrics units ( $\mathrm{m} / \mathrm{s}$ etc.).

These errors were avoided by re-calibrating the exterior orientation parameters for each scene. In such a calibration, prominent points in the camera image are marked pixel-accurately and their correspondence is indicated in the map (see Figure 4). From the point correspondences, the exterior orientation of the camera was calculated.

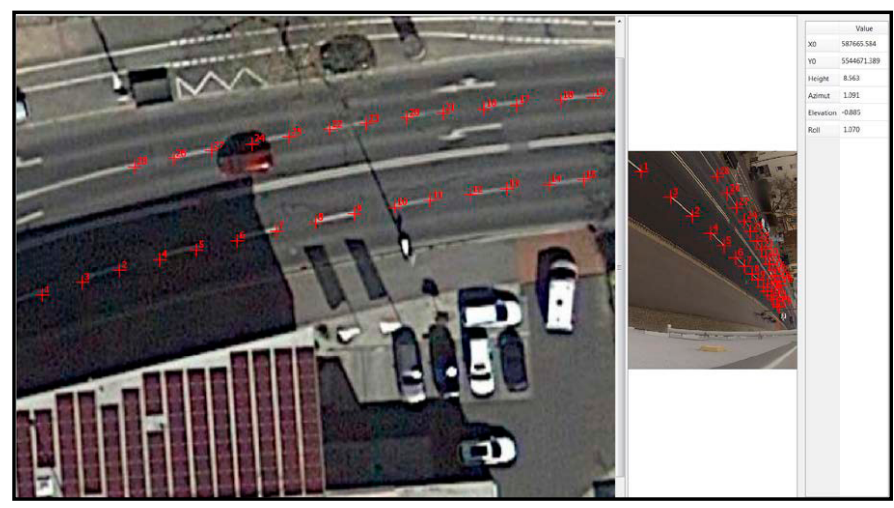

Figure 4. Corresponding points in map and camera image 
As a result, it was found that the accuracy of projecting the camera image onto the map image was better than $10 \mathrm{~cm}$ for the entire area of interestfrom the entrance of the road users to the exit of the rescue station. The calibration is considered suitable for carrying out the investigation (for more detailed consideration of the influence of measurement accuracy on the determination of critical situations in traffic see Leich et al. (2016).

\subsection{Providing Training Data for Virtual Loops}

During the automatic processing of the video data, the vehicles pass previously defined image areas (virtual loops). A pattern recognition algorithm determines how similar the image content is to the image of classified reference vehicles and outputs a corresponding similarity value. The Histogram of Oriented Gradients (HoG) combined with a support vector machine (SVM) was used as recognition algorithm as proposed by Dalal \& Triggs (2005).

In order to achieve optimum optical loop performance, training data was manually created over the entire 14-day period under various weather conditions (see Figure 5). The study generated approximately 3000 training data examples (50\% vehicles and 50\% background) for three virtual loops (right lane, lane changer and left lane) and finally estimated the detection quality based on samples from the various randomly selected scenes. Within the 14 days, out of 526 vehicles 524 were correctly recognized (true positive) having 5 errors (false positives). The pattern recognition algorithm and the number of training data were therefore considered suitable for carrying out the examination.

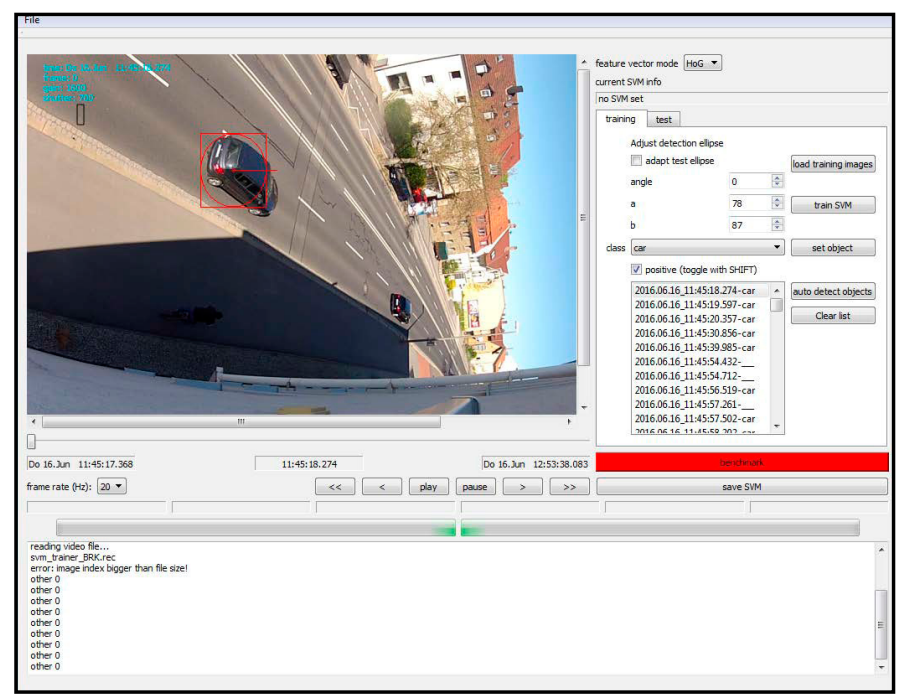

Figure 5. Graphical user interface for image processing methods training

\subsection{Image Processing and Calculation of Trajectory}

In the following the algorithm for object detection and vehicle tracking is briefly explained: The whole process of detecting a vehicle and tracking it, resulting in a trajectory, is automated. If a vehicle passes through the virtual loops (red ellipses), the calculated similarity score rise, as shown in Figure 6. There, only one MICU passes through the loops svm_2 (left lane) and svm_O (lane changer). The software generates the object candidate 7 and tracks it through the scene using an optical flow algorithm (see Leich et al. 2015). Then a red car passes through the virtual loops $s v m_{-} 1$ (right lane) and svm_O (lane changer). The loops svm_ 0 and svm_1 show increased similarity scores. The non-maximum suppression algorithm selects the object candidate 11 and tracks it. The projection onto the map (Figure 7) shows the objects 2 and 11 in $3 \mathrm{D}$ (or 2.5D) coordinates.

For all trajectories, space-time images, speedtime diagrams, and speed-location diagrams were generated. The diagrams were used to determine the following parameters:

- Entry speed into the detection area, directly readable in speed-time diagram

- Maximum deceleration, calculated by selecting two points in time in the velocity-time diagram

- Auxiliary variable: Time of braking (braking acceleration exceeds $1 \mathrm{~m} / \mathrm{s}^{2}$ ). This time was read from the acceleration time diagram.

- Location of the braking determined in the space-time image from the time of braking

- Location where the walking speed of $1.5 \mathrm{~m} / \mathrm{s}$ was reached. Readable in the speed-space diagram

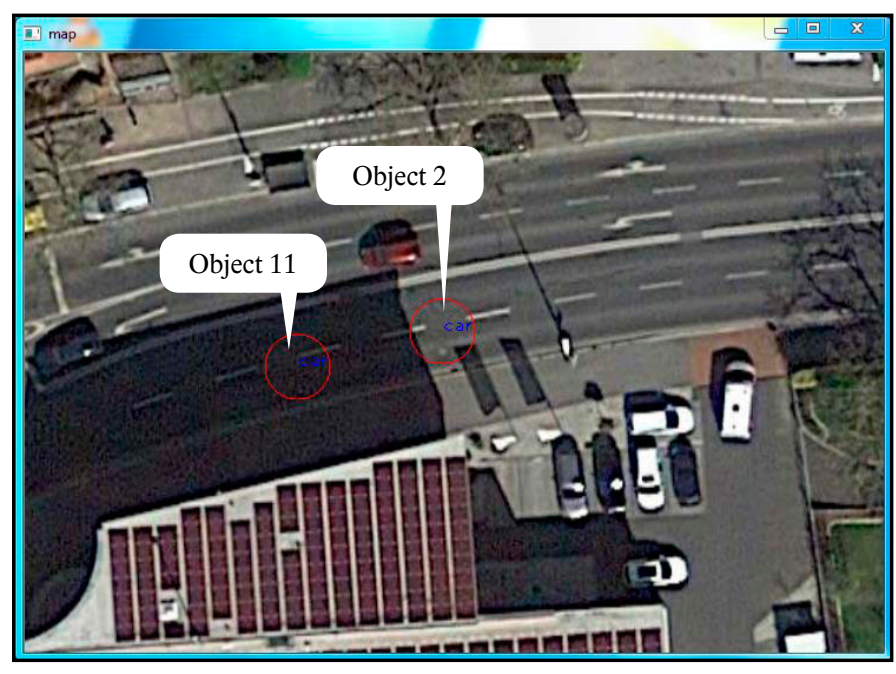

Figure 6. Object locations after perspective projection 

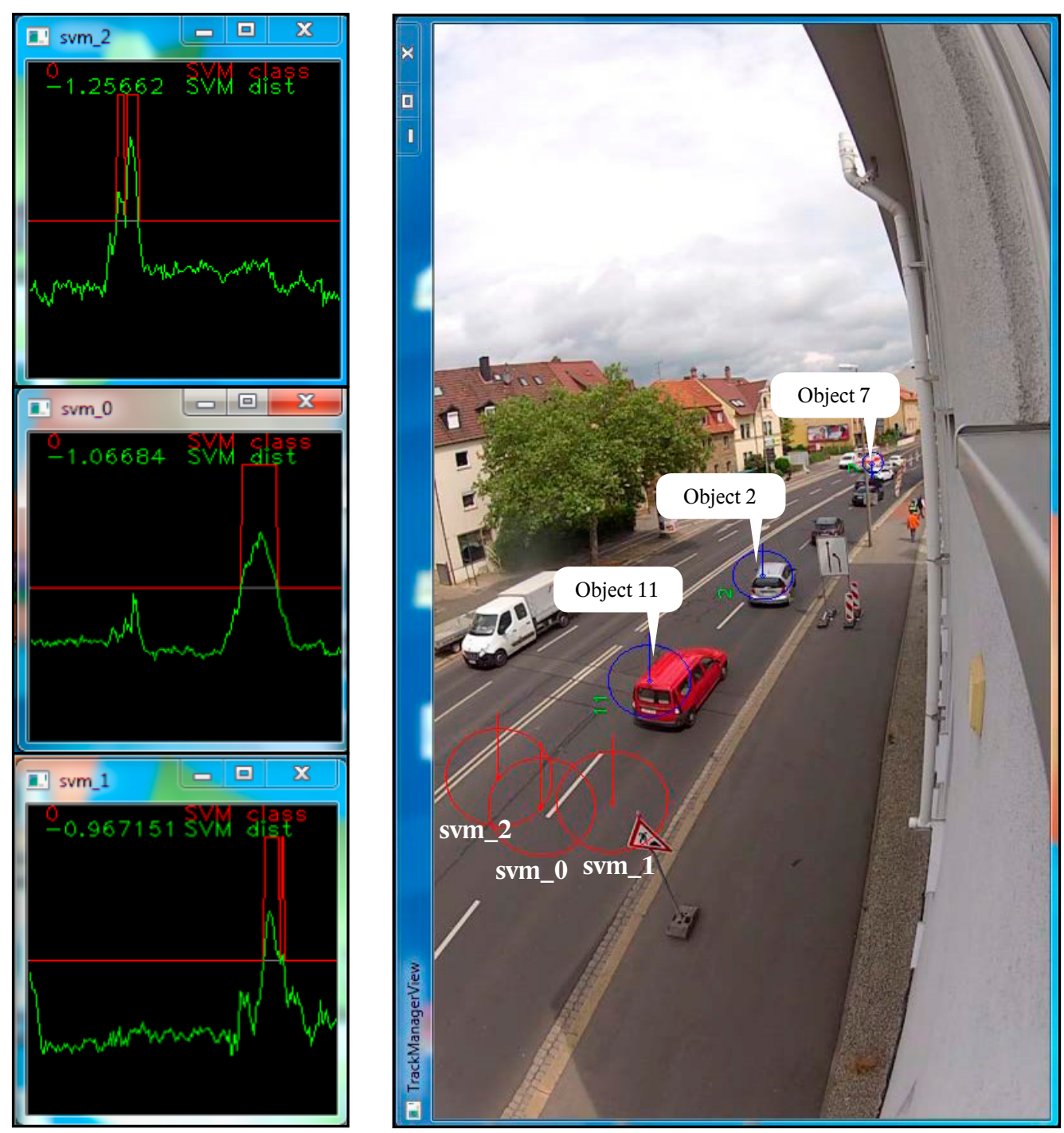

Figure 7. Three virtual loops and time plot of the similarity score (SVM_1: right loop, SVM_0: middle loop, SVM_2: left loop)

\section{RESULTS}

For the relevant indicators, distributions and averages were determined. See Figure 7 through Figure 10, Table 2 and Table 3. All calculated indicators show a tendency towards more favorable values in terms of criticality when the additional flash light is in use.

- The low entry speeds indicate that braking is often already done before entering the observation area. The average entry speed is $8.16 \mathrm{~m} / \mathrm{s}(29.4 \mathrm{~km} / \mathrm{h})$ with flash light vs. $9.22 \mathrm{~m} / \mathrm{s}(33.2 \mathrm{~km} / \mathrm{h})$ without flash light, or $12 \%$ lower.

The average of the maximum acceleration of the road users is $2.03 \mathrm{~m} / \mathrm{s}^{2}$ with side flash light than without side flash light $\left(2.18 \mathrm{~m} / \mathrm{s}^{2}\right)$.

- The distribution of brake application points over the path shows that vehicles start braking on average $3.5 \mathrm{~m}(8.1 \mathrm{~m}$ vs. $4.6 \mathrm{~m})$ earlier when the flash light is active. A vehicle approaching $50 \mathrm{~km} / \mathrm{h}$ slows down an average of 0.25 s earlier.

- The distribution of the brake application points (see Figure 9) shows that almost twothirds (64\%) of the brake application points are between 0 and $7 \mathrm{~m}$ when no side-marker lamp is active. The top of the histogram is in the interval $4 \mathrm{~m}-5 \mathrm{~m}$. With side flash light the top of the histogram is in the interval $0 \mathrm{~m}-1 \mathrm{~m}$ and 7 out of 11 (64\%) vehicles have already started to brake after $4 \mathrm{~m}$. This suggests that more road users start to brake earlier when the side flash light is active.

- When the side flash light is active, the vehicles reach walking speed after about $12 \mathrm{~m}$ on average, while this is the case after almost $16 \mathrm{~m}$ when no side flash light is active. The mean coincides with the top of the histogram (see Figure 10) 


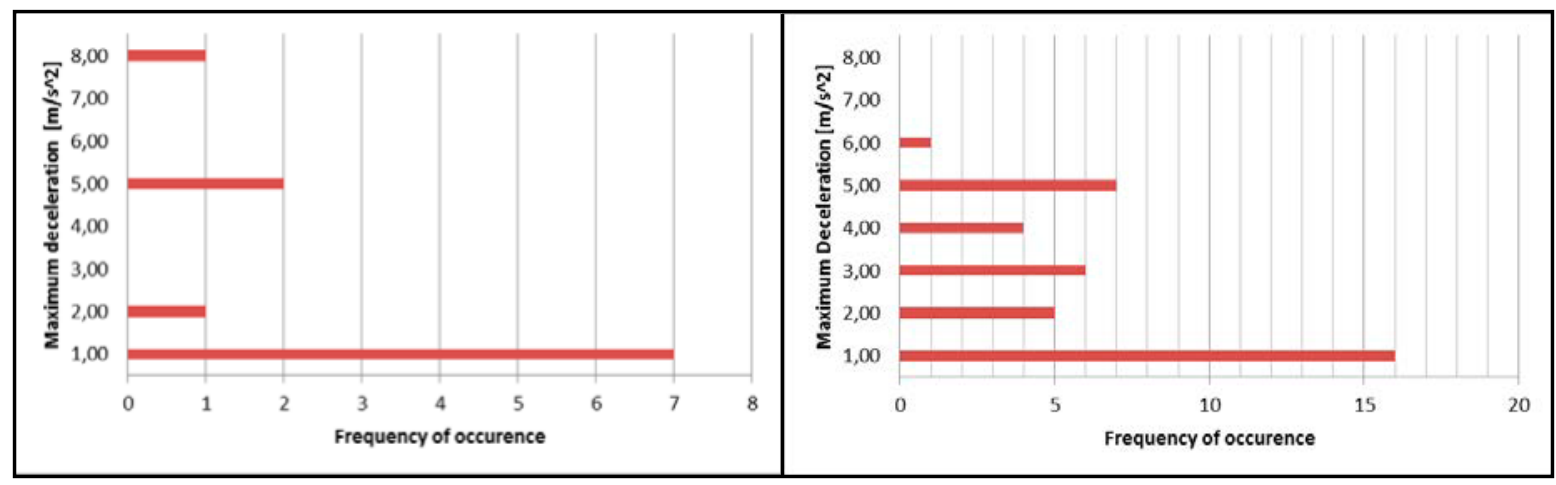

Figure 8. Histograms of the maximum deceleration in the detection area. Left with, right without flashlight

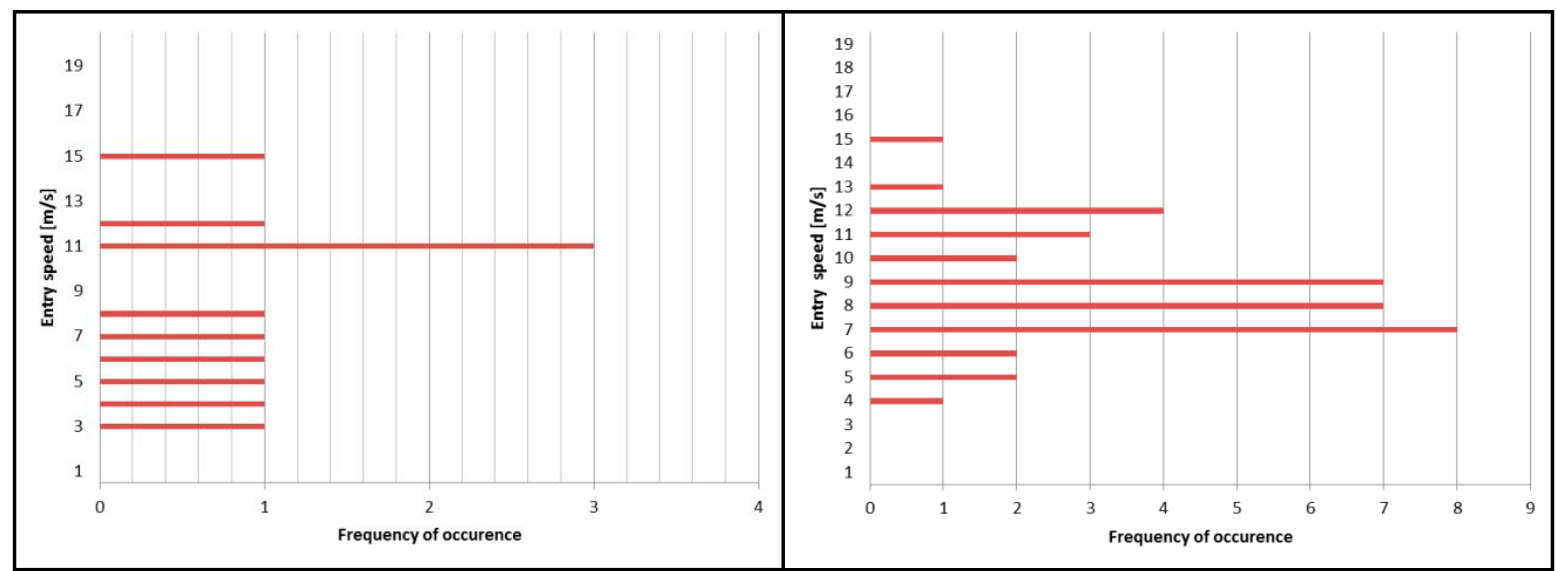

Figure 9. Histograms of the entry speeds into the detection area. Left with, right without flashlight

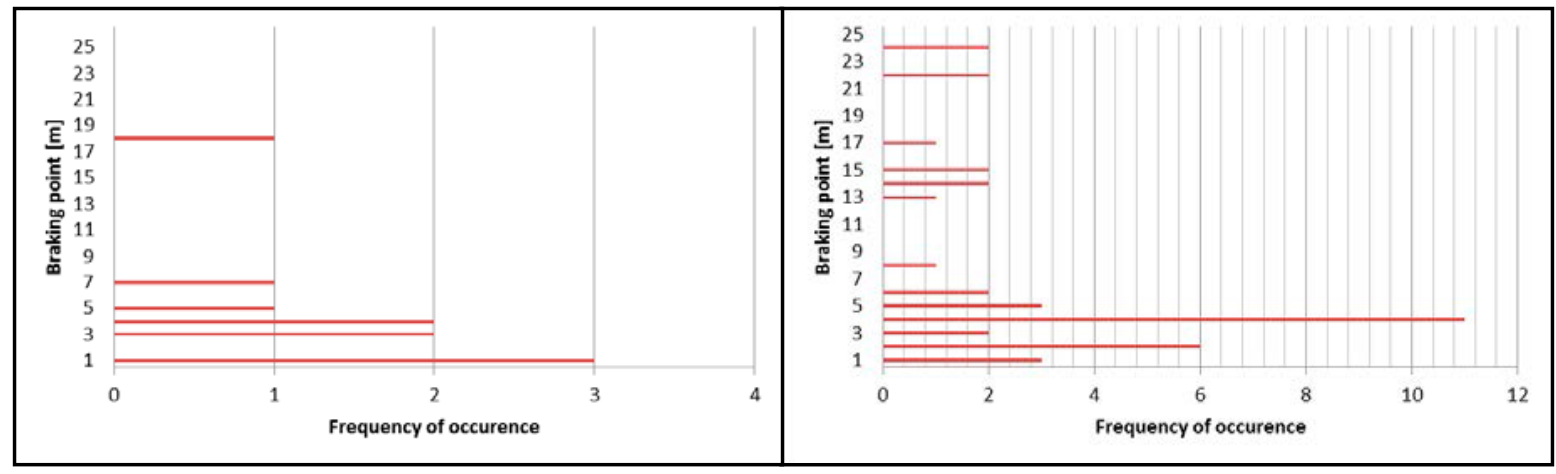

Figure 10. Histograms of braking points in the detection area. Left with, right without flashlight

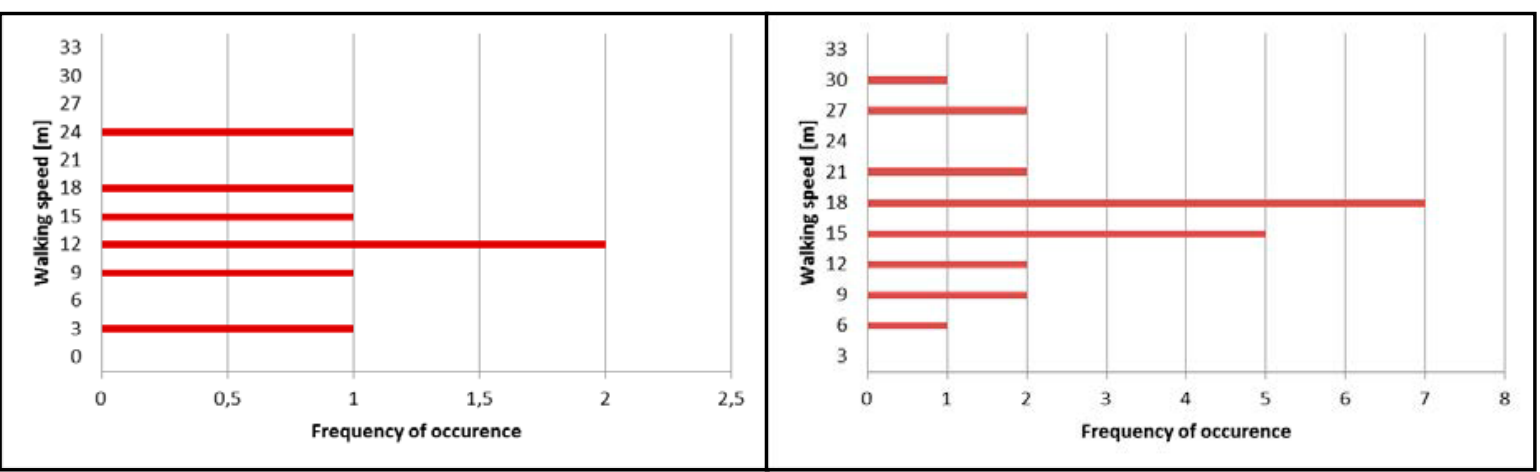

Figure 11. Histograms of the locations where the walking speed is reached in the detection area. Left with, right without flashlight 
For significance assessment of the results the Kolmogorow-Smirnow-Test (KS-Test) was conducted. This statistical test makes it possible to accept or reject a null hypothesis. The null hypothesis in this case is "There is no significant difference between the distribution of the entry speeds / maximum deceleration rates / braking points / points when the walking speed is reached with and without the use of additional flash lights". The null hypothesis is to be rejected if a maximum deviation (difference) $\mathrm{D}$ between the empirical frequency distributions exceeds a minimum value $\mathrm{D}_{\text {critical }}$.

Table 1 shows that the result is not significant for any of the parameters collected. The highest level is the significance level with only $9.5 \%$ error probability for the entry speeds, the lowest with $32 \%$ error probability for the distribution of the maximum deceleration. Illustratively, one can expect that on average of three comparable studies only one comes to the conclusion that no influence of the side flash light is detectable, while two confirm the influence. Conform to Bortz (2013), the rejection of the null hypothesis requires a significance level of $1 \%$ (99 out of 100 studies would give the same result).

Table 1. Significance of the results according to KSTest. Left column: distance of empirical frequency distributions, middle: critical distance, right: probability of error

\begin{tabular}{lccc}
\hline & $\mathrm{D}$ & $\mathrm{D}_{\text {critical }}$ & $\mathrm{P}$ \\
\hline Entry speed & 0,128 & 0,556 & $9,5 \%$ \\
Maximum deceleration & 0,223 & 0,556 & $32 \%$ \\
Braking point & 0,301 & 0,603 & $26 \%$ \\
Location of reaching walking speed & 0,35 & 0,704 & $25 \%$ \\
\hline
\end{tabular}

\subsection{Findings for Study Planning}

With the help of the KS-Test, it is possible to estimate how large the sample would have to be in order to achieve a reliable, statistically significant result. Here, the following assumptions are made: a) a measurement takes place over a longer period of time

b) again, trajectories are recorded by road users interacting with MICU

c) again, there are about four times more trajectories of duty rides without flash light than of service rides with flash light $\left(4 n_{\text {flash }}=n_{\text {noflash }}\right)$

d) the distance D between the empirical distributions always remains at the level measured in the pre-study

Then the number $n_{\text {flash }}$ of necessary trajectories can be calculated as follows:

$$
\begin{aligned}
& 4 n_{\text {flash }}=n_{\text {noflash }} \\
& D_{\text {critical }}=\lambda_{\alpha} \sqrt{\frac{n_{\text {flash }}+4 n_{\text {flash }}}{4 n_{\text {flash }} n_{\text {flash }}}}=\lambda_{\alpha} \sqrt{\frac{5}{4 n_{\text {flash }}}} \\
& n_{\text {flash }}=\frac{5}{4}\left(\frac{\lambda_{\alpha}}{D_{\text {critical }}}\right)
\end{aligned}
$$

The parameter $\lambda_{\alpha}$ is a constant of the KS-Test. For a significance level of $99 \%, \lambda_{\alpha}$ is 1.63 .

Table 4 summarizes the results of the calculations for the various parameters considered. It can be seen that the size of the sample would have to be increased by a factor of 20 in order to obtain a significant result at the entry speeds. This would mean that for 40 weeks (or less than a year) daily digital video would have to be recorded with a GoPro camera. For the other parameters, the estimated cost is 3 to 7 times the current 2 week recording time.

These estimates will only be confirmed if assumption d) is true. However, there is reason to believe that the distance between the distributions goes down when the number of data samples goes up. This is because sampling errors tend to be smaller if the empirical distributions are sampled on a finer grid. It is therefore advisable to provide a reserve.

Therefore, we assume that with a permanently installed camera in 24-hour operation, a collection time of 3-4 months should be sufficient for collecting enough data for significant results. 
Table 2: List of road users interacting with MICU with side flash light

\begin{tabular}{lllll}
\hline Data Base ID & v_0 $[\mathrm{m} / \mathrm{s}]$ & Max. Deceleration $\left[\mathrm{m} / \mathrm{s}^{\wedge} 2\right]$ & Braking point $[\mathrm{m}]$ & Walking speed $[\mathrm{m}]$ \\
\hline $\mathbf{7 0}$ & 6 & 1,68 & 2,5 & 9,5 \\
$\mathbf{7 2}$ & - & - & - & - \\
$\mathbf{7 3}$ & 5 & 0,29 & 1 & 8,5 \\
$\mathbf{7 4}$ & 2,5 & 0,50 & 1 & 2,5 \\
$\mathbf{7 5}$ & 10,7 & 0,60 & & \\
$\mathbf{7 6}$ & 10,5 & 4,67 & 18 & 23 \\
$\mathbf{7 7}$ & 11,7 & 5,00 & 3 & 14,5 \\
$\mathbf{7 9}$ & 3,7 & 0,63 & 1 & 9,5 \\
$\mathbf{8 2}$ & 10,5 & 0,75 & 5 & \\
$\mathbf{8 3}$ & 7,8 & 0,50 & 4 & 18 \\
$\mathbf{9 1}$ & 14,4 & 7,20 & 7 & 12,21 \\
$\mathbf{9 2}$ & 7 & 0,50 & 3,5 & \\
\hline Average & 8,16 & 2,03 & 4,60 & \\
\hline
\end{tabular}

Note: The vehicle with the ID 72 database (ID 7 in Figure 7) is not scored because it is an MICU

Table 3: List of road users interacting with MICU without side flash light

\begin{tabular}{|c|c|c|c|c|c|c|c|c|c|}
\hline $\begin{array}{l}\text { Data Base } \\
\text { ID }\end{array}$ & $\begin{array}{l}\mathrm{v} \_0 \\
{[\mathrm{~m} / \mathrm{s}]}\end{array}$ & $\begin{array}{l}\text { Max. } \\
\text { Decelaration } \\
{\left[\mathrm{m} / \mathrm{s}^{\wedge} 2\right]}\end{array}$ & $\begin{array}{l}\text { Breaking } \\
\text { Point }[\mathrm{m}]\end{array}$ & $\begin{array}{l}\text { Walking } \\
\text { speed [m] }\end{array}$ & $\begin{array}{l}\text { Data Base } \\
\text { ID }\end{array}$ & $\begin{array}{l}\mathrm{v} \_0 \\
{[\mathrm{~m} / \mathrm{s}]}\end{array}$ & $\begin{array}{l}\text { Max. } \\
\text { Decelaration } \\
{\left[\mathrm{m} / \mathrm{s}^{\wedge} 2\right]}\end{array}$ & $\begin{array}{l}\text { Breaking } \\
\text { Point }[\mathrm{m}]\end{array}$ & $\begin{array}{l}\text { Walking } \\
\text { speed }[\mathrm{m}]\end{array}$ \\
\hline 3 & 9 & 4,40 & 4 & 9,3 & 33 & 5,6 & 1,00 & 3 & 12 \\
\hline 4 & 9,7 & 0,50 & 5 & & 34 & 9,8 & 0,50 & 5 & \\
\hline 6 & 8,8 & 1,40 & 15 & 29 & 35 & 8,5 & 1,00 & 24,5 & \\
\hline 7 & 8,2 & 4,00 & 13,5 & 17 & 36 & 7,2 & 0,70 & 16 & 26 \\
\hline 8 & 7,5 & 2,22 & 8,5 & 8,5 & 37 & 7,6 & 1,70 & 15 & 20 \\
\hline 10 & 9,8 & 3,60 & 5 & & 39 & 15,2 & 0,50 & 7 & \\
\hline 11 & 4,5 & 2,00 & 2 & 3,5 & 42 & 12,6 & 2,60 & 23 & \\
\hline 13 & 9 & 0,20 & 23 & & 44 & 8,5 & 3,00 & 5 & \\
\hline 14 & 10,9 & 0,20 & 5,5 & 17 & 45 & 12,4 & 4,20 & 5 & 18 \\
\hline 15 & 11,4 & 4,40 & 5 & 18 & 46 & 10,8 & 0,40 & 5 & \\
\hline 17 & 9,2 & 3,00 & 4,5 & 14 & 49 & 12,4 & 0,50 & 3 & \\
\hline 20 & 9,6 & 4,40 & 4,5 & 12,5 & 50 & 6,2 & 1,20 & 4 & \\
\hline 21 & 13,4 & 6,00 & 7 & 17 & 54 & 5,2 & 0,10 & 2,5 & \\
\hline 22 & 7,4 & 2,61 & 3,5 & 9 & 57 & 7,2 & 1,00 & 2 & 14,5 \\
\hline 24 & 9,6 & 0,91 & 5 & & 60 & 12,4 & 4,70 & 15,5 & 18 \\
\hline 25 & 9 & 1,00 & 2,5 & & 62 & 10 & 4,00 & 25 & 27 \\
\hline 27 & 7,8 & 0,20 & 5 & & 63 & 9 & 4,00 & 18 & 21 \\
\hline 28 & 7,2 & 0,20 & 2 & & 64 & 7 & 2,00 & 3 & 14,5 \\
\hline 30 & 11,2 & 4,10 & 5,5 & 17 & 66 & 7,4 & 2,20 & 3 & 9,5 \\
\hline 32 & 11,2 & 4,20 & 5,5 & 14,5 & Mean & 9,22 & 2,18 & 8,10 & 15,95 \\
\hline
\end{tabular}


Table 4. Numbers of necessary trajectories for statistically significant results

\begin{tabular}{lll}
\hline & $\mathrm{D}$ & $n_{\text {flash }}$ \\
\hline Entry speed & 0,128 & 202 \\
Maximum deceleration & 0,223 & 67 \\
Braking point & 0,301 & 37 \\
Location of reaching walking speed & 0,35 & 27 \\
\hline
\end{tabular}

\section{DISCUSSION}

All the indicators strongly suggest that there is a safety benefit when using the additional side flash light. The vehicles brake on average $3.5 \mathrm{~m}$ earlier, they break less strongly, and they reach walking speed $4 \mathrm{~m}$ earlier. We conclude from this, that vehicles have earlier perception of the ambulance vehicle. Given an average local speed of $50 \mathrm{~km} / \mathrm{h}$ it can be assumed that the reaction time on the emergency vehicle is improved with a special signal of $0.2-0.3$ seconds. In the presence of the flash light, the vehicles drive on average slower in the detection area because they presumably brake before.

Reaching walking speed by an average of 4 mearlier can, in the case of an emergency, be decisive in determining whether or not an accident happens. As a result of this preliminary study, it is recommended to perform a complete study (phase 1), with a statistically significant underpinning of the result. It is very likely to be expected that robust test results and sufficient arguments for type approval of the additional side flash lights can be obtained.

The methods used are considered suitable for determining the ascertained parameters. In addition, it could be determined by visual inspection of the videos during the evaluation that the detection and tracking of road users succeeds sufficiently well.

Due to the fact that true interactions occurred only in comparatively few of the recorded scenes, it is possible that the result of this preliminary study is falsified by the influence of randomness; the safety gain could not be proven significantly due to the insufficient database. This means that another study over a similarly short period of time may yield different and even opposite results. For example, the greatest deceleration rate was registered with the ID 91 vehicle when an MICU with side flash light exited. A statistically reliable statement can only be made af- ter evaluating data from a larger sample of interactions. This would have to be collected over a longer observation period of 3 - 4 months.

It is desirable to use a fixed camera so that no daily recalibration (determination of the external orientation) is required. It is also desirable to include and evaluate the coverage area in the west direction (Figure 2). This seems sensible due to the large number of vehicles that already had started braking before reaching the observed region of interest. Likewise, an automated detection of the acoustic signaling of the MICU is desirable.

\section{CONCLUSIONS}

In this paper, a preliminary study on the safety impact of additional flashing blue lights was presented.

The method of the study is based on traffic surveillance data from a camera located at the road side. The camera's field of view covered the zone on a public road, where conflicts between MICU and everyday traffic were expected. The exit of the BRK station is dangerous, because leaving ambulance vehicles are hidden by the building. The building is a clear obstruction for the line of sight of normal vehicles approaching the conflict zone. Additional flash lights should enable approaching traffic to see the MICU about $1.5 \mathrm{~m}$ earlier than with solely standard flash lights on the roof of the vehicle.

The findings of the study are that vehicles enter the field of view at a lower speed, brake earlier, brake less sharp and reach walking pace $4 \mathrm{~m}$ earlier on average, when the additional blue lights are in use.

In order to obtain statistical significance a longer observation period of 3 - 4 months is needed. A review of the state of the art of surrogate safety studies suggests that a full study must consider more than one location for general conclusions.

The paper demonstrates, that it is possible to do this type of study with very little budget and very low hardware expenses. The expenses on hardware equipment were less than $500 €$ and the main part was the consumer action camera.

Acknowledgements: The study published in this paper was funded by the safety research section of the Bavarian Red Cross and the I.MoVe project within the German Aerospace Center. 


\section{REFERENCES}

Boone, C. M., Avery, L. W. and Malone, T. B. (2014): A Research Study of Ambulance Operations and Best Practice Considerations for Emergency Medical Services Personnel. First responders Group Department of Homeland Security Science \& Technology Directorate, December 10, 2014, retrieved from: https://www. naemt.org/docs/default-source/ems-health-and-safety-documents/health-safety-grid/ambulance-driver-(operator)-bestpractices-report.pdf?sfvrsn $=2$

Casey, C., Grant, P. E. and Merrifield, B.: Analysis of Ambulance Crash Data. Fire Protection Research Foundation report, Date of issue: September 2011

International Association of Fire Fighters (2010): Best Practices for Emergency Vehicle and Roadway Operations Safety in the Emergency Services. Retrieved from: http://www.iaff.org/ hs/evsp/best\%20practices.pdf

Gettman, D., Pu, L., Sayed, T., and Shelby, S.: Surrogate Safety Assessment Model and Validation: Final Report, Report No. FHWA-HRT-08-051, Turner-Fairbank Highway Research Center, Federal Highway Administration, McLean, VA, 2008. Retrieved from: http://www.fhwa.dot.gov/publications/research/safety/08051/index.cfm

Souleyrette, R., Hochstein, J.: Development of a Conflict Analysis Methodology Using SSAM. Final Report, Center for Transportation Research and Education, Iowa State University, 2012. http://www.intrans.iastate.edu/research/documents/researchreports/conflict_analysis_w_cvr.pdf (page 40-41)

Guo, F., Klauer, S. G., McGill M. T., and Dingus, T. A.: Evaluating the Relationship Between Near-Crashes and Crashes: Can Near-Crashes Serve as a Surrogate Safety Metric for Crashes? Report, U.S. Department of Transportation National Highway Traffic Safety Administration, October 2010

Ismail, K., Sayed, T., \& Saunier, N. (2010). Automated analysis of pedestrian-vehicle: conflicts context for before-and-after studies. Transportation Research Record: Journal of the Transportation Research Board, (2198), 52-64.

Fitzpatrick, K. (2000). Accident mitigation guide for congested rural two-lane highways (No. 440). Transportation Research Board.

Leich, A., Kendziorra, A., Saul, H., and Hoffmann, R. (2016). Calculation of Error Rates for Detection of Critical Situations in Road Traffic. In 95th TRB Annual Meeting-Compendium of Papers (Vol. 95). Transportation Research Board.

Dalal, N. \& Triggs, B. (2005) Histograms of oriented gradients for human detection, 2005 IEEE Computer Society Conference on Computer Vision and Pattern Recognition (CVPR'05), San Diego, CA, USA, 2005, pp. 886-893 vol. 1.

Leich, A., Junghans, M., Kozempel, K., \& Saul, H., Road user tracker based on robust regression with GNC and preconditioning. (2015) In SPIE/IS\&T Electronic Imaging (pp. 940702940702). International Society for Optics and Photonics.

Kolmogorov-Smirnov-Test. Retrieved from: https://en.wikipedia. org/wiki/Kolmogorov-Smirnov_test

Bortz, Jürgen: Lehrbuch der Statistik: Für Sozialwissenschaftler. Springer Verlag 2013 